\title{
ANALYSIS OF ADAPTATION CAPACITY IN THE CONTEXT OF BUILDING RESILIENCE TO THREATS- LOCAL LEVEL
}

\author{
Karina GÓRSKA-ROŻEJ, Marlena NIEMIEC \\ War Studies University, Warsaw, Poland \\ k.gorska@akademia.mil.pl; m.niemiec@akademia.mil.pl
}

\begin{abstract}
Adaptability is the base in the concept of adaptation, it activates social adaptation, have an impact on susceptibility reduction and on increasing of resilience to threats in communities at the local level. What is more, it allows to strengthen the economic opportunities of the society, uninterrupted social communication, social education process and social capital building, which is necessary in resilience building to threats. The subject of research in this article is adaptation capacities of the community at the local level. The main goal of the research was to identify the essence of adaptive abilities in building resilience to threats in communities at the local level. The research problem took the form of the following question: How important is adaptability in building resilience to threats in communities at the local level?
\end{abstract}

\section{Keywords: local communities, adaptation capacity, adaptation, resilience, threats}

\section{Introduction}

In this article, resilience is understood as the ability of society which is vulnerable to threats to adopt to a new situation by resisting or making changes in order to maintain an acceptable level of existence. The main purpose of development of building resilience to threats is to engage in local communities commitment to actions which reduce the risk of threats. Resilience has three main areas: the level of local community in aspect of self-organization; the amount of disruption that this community can endure and the level of local community in aspect of building and adaptation increasing and learning [1]. The base for increasing of the resilience level to threats in communities at the local level is their adaptation capacities. Its basic components include: adequate communication, high level of social capital and economic development [2]. The object of this article research is adaptation capacities of communities at the local level.
The main aim of research was to identify the essence of adaptability in building resilience to threats in communities at the local level. The research problem took the form of the following question: How important is adaptability in building resilience to threats in communities at the local level?

\section{How can we understand resilience to threats?}

This term was used formally for the first time in ecology (the 70s of 20th century) as one of the criteria of possibility of a specific system to accept changes and continuation of proper functioning. The roots of this term are also present in psychiatry, physics and psychology [3]. It was also used in the aspect of community safety management at the local level. The focus on the concept of resilience is the result of uncertainty increasing and changes in the nature of threats. Resilience means the ability of local communities to self- 
organize and adaptation to emerging circumstances [4]. Resilience is also the speed of local communities to return to its original state after the occurrence of a threat [5]. Resilience to threats can be also understood or translated in few aspects:

- natural one;

- physical one;

- institutional one;

- social one;

- economic one [6].

A resilient society understands and is aware of the threats to which is exposes, prepared for threats appearing and changes which occur while negative events occure. Therefore, resilience is associated with the ability to dealing with sudden and unexpected changes and smooth returning to normal functioning. To determinate the resilience of local community, it is necessary to diagnose and explore the level of preparation and abilities to respond to specific threats among members and the entire group. Resilience should increase with learning process, which appears after the emergence of threat. This process helps to increase of the adaptabilities of the local community and discussed resistance to further and potential threats.

\section{Adaptation and adaptability}

While analysing the literature of the subject, we can find various approaches to the concept of adaptation. In sciences about nature, it should be referred to the development of behavioural or genetic traits, thanks to which individual organisms have a chance to survive in the environment [7]. On the other hand, from the sociological point of view, adaptation should be understood as behavioural, cultural and technological adjust of individuals, social groups or institutions to changes that take place in the environment [8]. Adaptation is perceived as adjusting the behaviours and characteristics of a system in order to increase its ability to cope with external threats, it allows to reduce the risk associated with these threats by reducing social susceptibility [9]. Adaptation is part of resilience and includes the ability to adapt to changing external and internal conditions, thus it allows to develop with existing trends [10]. Social adaptation is activated with the help of adaptive abilities. They contribute to increasing resilience and reducing susceptibility in local communities. Adaptability is also related directly to efficient and effective management of the safety of local communities because it allows individuals and groups to adapt to potential threats, concerns on changes in the administrative structures of local community management, allows for adequate social communication, social education process, social capital building and strengthening the economic possibilities of the society. In connection with that, it is worth listing the components of the adaptability, which include:

- proper communication (quantity and quality of transmitted and collected information, reducing chaos and uncertainty, feedback, information updating);

- high level of social capital (cooperation, empathy, contacts establishing and maintaining, social support, attachment to the place);

- interpersonal competences (capacity to receive criticism, creativity, partnership, solving problems together, leadership, empathy);

- economic development (high level of economic resources, equal access to economic resources) [11].

In the modern approach in building resilience to threats in local communities, the improvement of the previously mentioned adaptive abilities is associated with the activities such as:

- developing of organizational networks. Created networks, established interpersonal relationships and friendships are the basis of an immediate response to threats. What is more, they provide opportunities to decide fast on activities to be carried out as well as 
organization of resources and the plan for their use;

- universal access to social capital, mutual relations and trust of individuals. The inclusion of the local community in the processes of planning and preparing tasks that are directly concerned on that community, gives each individual an access to the described capital. Thanks to this, the resilience of the local community grows, as its level of autarkism increases,

- support for social initiatives to improve safety, e.g initiative to eliminating crime - implement of monitoring system in places where the most events like this were recorded or where the community is afraid to be/fell anxious;

- obtaining information and building communication networks (paying attention to access to local knowledge and skills, disseminating information, planning and implementing training for the local community, social culture building);

- expanding and modernizing the economic resources of society, which determines minimizing the risk of destructive phenomena and improving the methods of their dissemination. It worth's considering the construction of economic diversity, thanks to which the most needy people will have a direct access to resources when an event occurs. In such a situation, responsible management of resources, creative problem solving and implementation of political actions which are concerned on social good, is extraordinary in shaping the resilience of local communities [12].

Access to local knowledge and skills is related to equipping people with specific skills, which become useful in responding to threats or selecting people from the local community who already have such skills (such as: knowledge of topography and indication of movement routers, the ability to carry out and coordinate the evacuation of people from endangered areas to safe ones, leadership skills, the ability to provide psychological or medical assistance). The dissemination process can start from e.g. creating information platform, which will include instructions on the actions to be carried out in order to avoid the threat individually or in a group or minimize its negative effects; information about sources of financing in case of losses incurred as a result of negative events. It is important that the mentioned area of data exchange is promoted in the media - appropriate marketing activities should be implemented at the beginning, so it will disseminate it to the local community and constantly update it. The authors of such a platform should be aware that they are creating it in purpose to increase the resistance of the community to threats. They do it mostly by disseminating substantive and necessary information. Conducting training about potential threats, making the local community aware, sensitizing each individual to specific elements of social coexistence, increasing of vigilance and interest in what is happening around, in large extent shapes on the resilience of the local community and (as a result) allows for an efficient process of adaptation in case of changes that occur in the environment. The last important element in improving adaptation abilities is building a social culture. It is obvious that the process of building this culture should take place in terms of increasing resistance to threats, not (as often happens) on the constancy belief that "this problem does not concern us or will never do". Resilience should be increased through a properly planned and organized process of learning (with access to the necessary resources), understanding the threats, anticipating, being vigilant (individually and in groups) and thanks to the ability to adapt to new conditions.

\section{Constraints and factors that shape the adaptability of communities at the local level}

In this part of the article, it is reasonable to 
point that ability to adapt to changing condition of environment is unfortunately limited by many aspects, such as:

- financial conditions;

- physical conditions;

- social conditions;

- legal and political conditions;

- administrative conditions [13].

Financial conditions are related to the wealth of individual wallets, financial capacity of individuals and the entire local community. This type of determinants is mainly considered in the context of coping when a threat occurs or while preparing for its appearance. Physical conditions are related to quality and quantity of infrastructure and its susceptibility to threats, as well as refer to the conditions of environment in which local community works. Social conditions imply the level of interpersonal relations as well as its quality and duration, the level of individuals trust to each other and the ability to cooperate. Political conditions model the functioning of local communities and shape the level of their adaptation abilities, which these communities do not have any influence on (the only interference by the society may be high electoral turnout during local elections). Administrative conditions are related to political conditions which are concerned on relations with local authorities and their activity or passivity. It is worth to mention that the level of development of the region in which they live, has an impact on numerous limitations of the local communities adaptability. Limitations are the result of wrong relations between the government and the local community and also depend on the individual characteristics of institutions which are able to undertake various activities, focused on increasing of adaptability in a region, such as: encouraging the local communicate to participate in making decisions about local matters; making some decisions together with the residents; increasing the possibility of learning from mistakes. The factors that influence on the shape of adaptive abilities can be divided into: strategic and operational [14]. The last ones are considered as so-called hard and technical factors. They refer to long-term planning, which translates into efficient and effective implementation of tasks in the event of a threat. What is more, attention is paid to the role of leadership in a group action, division of labour, the ability to communicate and obtain information. Strategic factors are related to soft aspect, such as: knowledge, social culture, organizational culture, attitudes and values of local community members, social relations, relations with local authorities. In this connection, it is reasonable to say that adaptive abilities are used to build resilience to threats in local communities to strengthen the ability to respond flexibly to changes in the environment. It is adaptation that relates to those changes that have an impact on the society survival in new conditions in the longer term.

\section{Conclusions}

Local communities activities which have adaptive nature may be carried out in a preemptive or passive, unitary or planned way. Current approach to generally understood management of community safety is focused on the planned, pre-emptive and innovative adaptation. Thanks to such actions, it is possible to reduce the susceptibility to unwanted imputes and increase of the immunity (of the title). From the analyze of the literature and referring to the content in this article, it should be stated that the primary aim of adaptive abilities in building resilience to threats in local communities is to raise the level of the ability to flexible respond to numerous changes and distortions in the functioning environment. Summing up, it is worth to point the elementary actions which can increase or maintain, on the acceptable level, the adaptability of local communities. The authors of the article pay attention to cooperation in this aspect (involvement of 
all local community members in group communities for the common good), social dialogue (information obtaining and sharing, sharing ideas, looking for a practical consensus together and making decisions accepted by the local community) and education (understanding of the nature and size of threats, social awareness of threats, access to programs that raise public awareness, access to the Internet and other media, education programs for children and teenagers which take into identification of threats and ways of behaving in case of occurrence/identification of threat). From the long-term observation of the Polish education systems, it should be concluded that it is not conductive to the mentioned elements, because children and adolescents are directed to rivalry in social relations, not in cooperation, as it is important building resilience to threats.

\section{References List}

[1] Carpenter S., Walker B., Anderies J. M., Abel N. From metaphor to measurement: resilience of what to what? Ecosystems. 2001; 4(8): 766-779.

[2] Norris F. H., Stevens S. P., Pfefferbaum B., Wyche K. F. Community Resilience as a Metaphor, Theory, Set of Capacities, and Strategy for Disaster Readiness. American Journal of Community Psychology. 2008; 41: 136-137.

[3] Holling C. S. Resilience and stability of ecological systems. Annual Review of Ecology and Systematics. 1973; 4: 2-19.

[4] Adger W. N. Vulnerability. Global Environmental Change. 2006; 16: 268-275.

[5] Pimm S. L. The complexity and stability of ecosystems. Nature 307. 1984; 321-324.

[6] Manyena S. B. The concept of resilience revisited. Disasters. 2006; 30(4): 440-449.

[7] N. Wolański, Ekologia człowieka. Ewolucja i dostosowanie biokulturowe. Warszawa: PWN; 2020.

[8] Smit B. Adaptation, adaptive capacity and vulnerability. Global Environmental Change. 2006;16(3): 283-284.

[9] Berkers F., Young O. The globalization of socio-ecological systems: An agenda for scientific research. Global Environmental Change. 2006; 16(3):304-314.

[10] Folke C., Carpenter S. R., Walker B., Scheffer M. Resilience thinking:integrating resilience, adaptability and transformability. Ecology and Society. 2010; 16(3):20-21.

[11] Norris F. H., Stevens S. P., Pfefferbaum B., Wyche K. F. Community Resilience as a Metaphor, Theory, Set of Capacities, and Strategy for Disaster Readiness. American Journal of Community Psychology. 2008; 41: 136.

[12] Norris F. H., Stevens S. P., Pfefferbaum B., Wyche K. F. Community Resilience as a Metaphor, Theory, Set of Capacities, and Strategy for Disaster Readiness. American Journal of Community Psychology. 2008; 41: 142-145.

[13] Birkmann J. First- and second- order adaptation to natural hazards and extreme events in the context of climate change. Natural Hazards. 2011; 58(2): 814.

[14] Armitage D. Adaptive Capacity and Community-Based Natural Resource Management. Environmental Management. 2005; 35(6): 707-708. 